\title{
Editorial
}

\section{Catheter ablation of ventricular tachycardia: are there limits?}

Of the numerous non-surgical therapies available to treat ventricular tachycardia (VT), catheter ablation is the least practised but has great promise for increased use in the treatment of this difficult arrhythmia. The paper by Furniss and colleagues ${ }^{1}$ in this issue of Heart describes the successful application of catheter ablation to a population of patients hitherto regarded as being virtually untreatable in this way; those whose arrhythmia leads quickly to collapse. Is their experience reproducible? If so, does it represent a new limit for the technique or does it offer a glimmer of hope that all ventricular tachyarrhythmias, including ventricular fibrillation, might be treatable by catheter ablation?

Catheter ablation has become the treatment of choice for most supraventricular tachycardias (SVTs) with the sole exception, for the moment, of atrial fibrillation. This has occurred because of the treatment's efficacy (a success rate of at least $90 \%$ ) and safety. Currently, when catheter ablation is applied to treat ventricular tachycardia (VT), the aim is to improve quality of life but not prognosis. This is so whether VT arises benignly within a normal heart or in the context of structural heart disease, when the patient's prognosis is ultimately determined by the severity of the underlying disease. In the former, success rates approaching those for ablation of accessory atrioventricular pathways can be anticipated ${ }^{2-7}$ so that for many, usually young patients, ablation may, as with SVT, be the treatment of choice.

In patients with VT complicating structural heart disease, the complexity of the target arrhythmia substrate has so far limited the role of catheter ablation. Despite this, in this patient group there is an established and increasingly important role for catheter ablation in treating VT, which is when the arrhythmia triggers unacceptably frequent therapy from an implanted cardioverter defibrillator (ICD). Such tachycardias are typically relatively slow, often as a result of antiarrhythmic drug treatment prescribed to reduce their incidence. The tachycardias are therefore usually well tolerated. This facilitates the mapping of complex VT substrates so that the culprit VT may be successfully ablated and the frequency of device therapy thereby reduced. ${ }^{89}$ The aim of this therapeutic approach is to reduce ICD therapy to tolerable levels and accept that the VTs not ablated will recur (less frequently) and be terminated safely by the ICD. This compromise is accepted because of the high risk and failure rates of antiarrhythmic surgery for VT that might achieve more complete ablation of multiple VTs.

The observation that catheter ablation may reduce the requirement for ICD therapy has encouraged the wider application of catheter ablation to treat patients with structural heart disease and VT. To achieve this, two important limitations, which have hitherto contributed to low success and high recurrence rates in this patient group, must be overcome. These are: firstly, the time required for precise localisation of the correct target for ablation (mapping); secondly, the small size of the lesions that are created when using radiofrequency $(\mathrm{RF})$ energy.

If ablation $i$ to be applied more widely to such patients, how are these limitations to be overcome? For the creation of controllable larger ablation lesions, cooled tipped RF delivery has already been shown to be effective ${ }^{10}$ while alternative forms of energy delivery capable of creating large lesions, such as ultrasound, laser, microwave and percutaneous cryotherapy, are at an early stage of development.

\section{New mapping tools}

The limitations of creating small lesions are greatest when targeting arrhythmias where mapping is difficult, namely multiple or short-lived arrhythmias which cause collapse, and are most frequently encountered when VT complicates structural heart disease. New mapping tools have been developed, which either provide global mapping data (noncontact and basket mapping techniques) ${ }^{11}{ }^{12}$ or create maps that correlate anatomy with electrical activation (Carto, Localisa, Realtime Position Management). ${ }^{13-15}$ Initial experience with the non-contact mapping system used to direct standard RF catheter ablation of the complex substrates of patients with multiple VTs has been encouraging. The requirements for ICD therapy were reduced and recurrence of VT in patients was rare. ${ }^{11}$ With global mapping methods, substrates for short lived and multiple arrhythmias can be defined, the data stored, and an ablation catheter navigated to the chosen site(s) enabling ablation of the target either during sinus rhythm or at the onset of a VT that would otherwise be of short duration. This has been confirmed by the experience using non-contact mapping when all target and many other VTs were successfully ablated. ${ }^{11}$ Approximately half of the energy applications in that series were delivered during sinus rhythm to sites that had been identified during VT as being crucial to supporting tachycardia. ${ }^{11}$

Furniss and colleagues ${ }^{1}$ describe the successful ablation of patients in whom VT had, of necessity, to be of short duration. If their experience could be repeated, are the new mapping techniques therefore to be of no additional value in treating these patients? Furniss and colleagues employed an "in-house" anatomical mapping process to help define the scar of myocardial infarction similar to an approach taken using the Carto mapping system where endocardial areas generating low voltages have been labelled as infarct scars. ${ }^{16}$ They then delivered RF energy to create linear lesions across scar boundary. In some of their patients, the linear lesions connected inferior infarct scar to the posterior mitral valve annulus, creating an "isthmus" ablation as described by Wilber and associates. ${ }^{17}$ However, several of their patients had suffered anterior myocardial infarctions and did not conform to that group.

\section{Anatomical guided approach}

That Furniss and colleagues were successful in treating their patients represents the most intriguing and important, but also frustrating, aspect of their paper. ${ }^{1}$ Their success is encouraging, but because of the predominantly anatomical guided approach (rather than electrophysiologically guided) adopted to the ablations, we cannot evaluate how the lesions modified the arrhythmogenic substrates. The authors employed pace mapping to help define the correct ablation sites. However, they applied this during sinus rhythm. The process by which paroxysmal VT is generated in patients 
with structural heart disease usually involves the development of an area of "functional" block to conduction. Such "functional" block is not fixed, not anatomical, and variable in its extent. When formed, it combines with an area of "fixed" conduction block caused by, for example, the scar of myocardial infarction, to create a protected channel for conduction that allows re-entry to occur. It therefore follows that in structural heart disease in sinus rhythm when there is no functional block, pacing, even from the VT substrate, generates a different ventricular depolarisation sequence and therefore a different QRS morphology from that produced by VT. Despite this, Furniss and colleagues performed pace mapping during sinus rhythm to match the ECG appearance of VT. Perhaps it was because these investigators did not require a completely accurate match of the paced 12 lead QRS morphology with the QRS morphology generated by VT, and were using this technique only as a "rough" guide to location, that they were able to glean sufficient information from these manoeuvres to find successful ablation sites.

If catheter ablation is to advance to treat life threatening ventricular arrhythmias, progress such as that reported in this study will need to be fully understood. It should therefore be repeated when detailed global mapping information can add such understanding. We might then begin to imagine a day when catheter ablation can be deployed to so change arrhythmogenic substrates in patients with structural heart disease that it will improve their prognosis as well as their life's quality.

St Mary's Hospital \& Imperial College

London, UK

1 Furniss SS, Anil-Kumar R, Bourke JP, et al. Radiofrequency ablation of haemodynamically unstable ventricular tachycardia after myocardial infarction. Heart 2000;84:648-52.
2 Morady F, Kadish AH, DiCarlo L, et al. Long-term results of catheter ablation of idiopathic right ventricular tachycardia. Circulation 1990;82:2093-9.

3 Klein LS, Miles WM, Hackett FK, et al. Catheter ablation of ventricular tachycardia using radiofrequency techniques in patients without structural heart disease. Herz 1992;17:179-89.

4 Calkins H, Kalbfleisch SJ, el-Atassi R, et al. Relation between efficacy of radiofrequency catheter ablation and site of origin of idiopathic ventricular tachycardia. Am 7 Cardiol 1993;71:827-33.

5 Vohra J, Shah A, Hua W, et al. Radiofrequency ablation of idiopathic ventricular tachycardia. Aust N Z F Med 1996;2:186-94.

6 Rodriguez LM, Smeets JL, Timmermans C, et al. Predictors for successful ablation of right- and left-sided idiopathic ventricular tachycardia. $A m \mathcal{F}$ Cardiol 1997;79:309-14.

7 Chinushi M, Aizawa Y, Takahashi K, et al. Radiofrequency catheter ablation for idiopathic right ventricular tachycardia with special reference to morphological variation and long term outcome. Heart 1997;78:255-61.

8 Willems S, Borggrefe M, Shenasa M, et al. Radiofrequency catheter ablation of ventricular tachycardia following implantation of an automatic cardioverter defibrillator. Pacing Clin Electrophysiol 1993;16:1684-92.

9 Strickberger SA, Man KC, Doaud EG, et al. A prospective evaluation of catheter ablation of ventricular tachycardia as adjuvant therapy in patients with coronary artery disease and an implantable cardioverter-defibrillator. Circulation 1997;96:1525-31.

10 Skrumeda LL, Mehra R. Comparison of standard and irrigated radiofrequency ablation in the canine ventricle. F Cardiovasc Electrophysiol 1998;9: 1196-205.

11 Schilling RJ, Peters NS, Davies DW. Feasibility of a noncontact catheter for endocardial mapping of human ventricular tachycardia. Circulation 1999;99:2543-52.

12 Schalij MJ, van Rugge FP, Siezenga $M$, et al. Endocardial activation mapping of ventricular tachycardia in patients. First application of a 32-site bipolar mapping electrode catheter. Circulation 1998;98:2168-79.

13 Gepstein L, Hayam G, Ben-Haim S. A novel method for nonfluoroscopic catheter-based electroanatomical mapping of the heart. Circulation 1997;95:1611-22.

14 Wittkampf FHM, Wever EFD, Dersken R, et al. New technique for real-time 3-dimensional localization of regular intracardiac electrodes. Circulation 1999;99:1312-17.

15 De Groot NMS, Bootsma M, Schalij MJ. Radiofrequency catheter ablation of atrial flutter guided by a new 3-D signal based tracking system: first clinical results [abstract]. PACE 2000;23(II):579.

16 Callans DJ, Ren J-F, Michele J, et al. Electroanatomic left ventricular mapping in the porcine model of healed anterior myocardial infarction; correlation with intracardiac echocardiography and pathological analysis. Circulation 1999;100:1744-50.

17 Wilber DJ, Kopp DE, Glascock DN, et al. Catheter ablation of the mitral isthmus for ventricular tachycardia associated with inferior infarction. Circulation 1995;92:3481-9.

\section{Rapid viewpoints}

Welcome to this new feature for Heart Online (www.heartinl.com) where we welcome your comments on topical issues in cardiology. Initially, views on the recent publication of the [National Institute for Clinical Excellence] NICE guidelines on IIb/IIIa inhibitors ${ }^{\star}$ are encouraged. These have produced many different reactions, and a certain amount of surprise.

If you want to comment on these guidelines, or other matters, please access this item on the Heart web site (www.heartjnl.com/cgi/content/full/84/6/586) and use the Rapid Response facility (click on eLetters: Submit a response to this article). Please limit your views to 750 words and 5 references, shorter if possible (sorry but we cannot accept images with this facility). Your views will be posted on Heart Online within a few days subject to editorial screening.

ROGER HALL Editor

${ }^{\star} \mathrm{A}$ pdf version of "Guidance on the use of glycoprotein IIb/IIIa inhibitors in the treatment of acute coronary syndromes" (September 2000) is available from the NICE web site (www.nice.org.uk/nice-web/pdf/ Nice+GLYCOPROTEIN+12+guidan.pdf) 